\title{
SUPERHARMONIC FUNCTIONS IN A DOMAIN OF A RIEMANN SURFACE
}

\author{
ZENJIRO KURAMOCHI
}

To Professor Kiyoshi Noshiro on the occasion of his 60th birthday

Let $R$ be a Riemann surface. Let $G$ be a domain in $R$ with relative boundary $\partial G$ of positive capacity. Let $U(z)$ be a positive superharmonic function in $G$ such that the Dirichlet integral $D(\min (M, U(z)))<\infty$ for every $M$. Let $D$ be a compact domain in $G$. Let ${ }_{D} U^{M}(z)$ be the lower envelope of superharmonic functions $\left\{U_{n}(z)\right\}$ such that $U_{n}(z) \geqq \min (M, U(z))$ on $D+\partial G$ except a set of capacity zero, $U_{n}(z)$ is harmonic in $G-D$ and $U_{n}(z)$ has M.D.I. (minimal Dirichlet integral $) \leqq D(\min (M, U(z)))<\infty$ over $G-D$ with the same value as $U_{n}(z)$ on $\partial G+\partial D$. Then ${ }_{D} U^{M}(z)$ is uniquely determined. Put ${ }_{D} U(z)=\lim _{M=\infty}{ }_{D} U^{M}(z)$. The mapping from $U(z)$ to ${ }_{D} U(z)$ is clearly linear.

Hence there exists a positive measure $\lambda(\xi, z)^{[1]}$ such that ${ }_{D} U(z)=\int U(\xi) d \lambda(\xi, z)$ for $z \in G-D$. If for any compact domain $D,{ }_{D} U(z)=U(z)$ or ${ }_{D} U(z) \leqq U(z)$, we call $U(z)$ a full harmonic (F.H.) or full superharmonic (F.S.H.) function in $G$ respectively. If $U(z)$ is an F.S.H. in $G$ and $U(z)=0$ on $\partial G$ except at most a set of capacity zero, $U(z)$ is called an $\mathrm{F}_{0}$. S.H. in $G$. Let $U(z)$ be an F.S.H. in $G$. Then ${ }_{D} U(z) \uparrow$ as $D \uparrow$. Put ${ }_{D} U(z)=\lim _{n}{ }_{D \cap G_{n}} U(z)$ for a non compact domain $D$, where $\left\{G_{n}\right\}$ is an exhaustion of $G$ with compact relative boundary $\partial G_{n}(n=0,1, \ldots)$.

Functiontheoretic mass $\mathfrak{M}^{f}(U(z))$ of an $F_{0} . S . H$. in $G$. Let $U(z)$ be an $\mathbf{F}_{0}$. S.H. in $G$. Then $g_{M}=E[z: U(z)>M]$ is open. Let $\omega\left(g_{M}, z, G\right)$ be a function in $G$ such that $\omega\left(g_{M}, z, G\right)$ is harmonic in $G-g_{M},=1$ in $g_{M}$ and has M.D.I. over $G-g_{M}$ and further $\omega\left(g_{M}, z, G\right)=0$ on $\partial G,=1$ on $\partial g_{M}$ except a set of cap. zero. Clearly such a function exists by $D(\min (U(z), M)))<\infty$ and $\min (M, U(z))=M$ on $\partial g_{M},=0$ on $\partial G$ except a set of cap. zero. It is easily seen, $\omega_{n}(z) \rightarrow \omega\left(g_{M}, z, G\right)$ in mean as $n \rightarrow \infty$, where $\omega_{n}(z)$ is a harmonic function in $R_{n} \cap\left(G-g_{M}\right)$ such that $\omega_{n}(z)=0$ on $\partial G, \omega_{n}(z)=1$ on $\partial g_{M}$ except a set of

Received July 12, 1966. 
capacity zero and $\frac{\partial}{\partial n} \omega_{n}(z)=0$ on $\left(G-g_{M}\right) \cap \partial R_{n}$, where $\left\{R_{n}\right\}$ is an exhaustion of $R$ with compact relative boundary $\partial R_{n}$. We call $\omega\left(g_{M}, z, G\right)$ C.P. (capacitary potential) of $g_{M}$ relative to $G$ and define $\operatorname{Cap}\left(g_{M}\right)$ by $D\left(\omega\left(g_{M}, z, G\right)\right)$. Then there exists a regular niveau ${ }^{[2]} C_{\delta}$ such that

$$
D\left(\omega\left(g_{M}, z, G\right)\right)=\int_{C_{\delta}} \frac{\partial}{\partial n} \omega\left(g_{M}, z, G\right) d s
$$

for almost $\delta$ with $0 \leqq \delta \leqq 1$.

Since $U(z)$ is an $\mathrm{F}_{0} . \mathrm{S}$.H. in $G, U(z) \geqq g_{M_{1}} U(z)$, whence

$$
E\left[z: g_{M_{1}} U(z)>M_{2}\right]=g^{\prime}{ }_{M_{2}} \subset g_{M_{2}}=E\left[z: U(z)>M_{2}\right] \text { for } M_{2}<M_{1} \text {. }
$$

By the definition $g_{M} U(z)=M \omega\left(g_{M}, z, G\right)$ in $C g_{M}$. On the other hand, $\delta \omega\left(g_{\delta}, z, G\right)$ $=\omega\left(g_{M}, z, G\right)$ in $C g_{\delta}$, where $g_{\delta}=E\left[z: \omega\left(g_{M}, z, G^{[3]}\right)>\delta\right]$ and

$$
D\left(\omega\left(g_{\delta}, z, G\right)\right)=\frac{1}{\delta} D\left(\omega\left(g_{M}, z, G\right)\right) \quad \text { for any } \delta<1 .
$$

Let $M_{1}>M_{2}$. Then by (1) and (2)

$$
\underset{c g_{M_{2}}}{D}\left(g_{M_{2}} U(z)\right)=M_{2}^{2} D\left(\omega\left(g_{M_{2}}, z, G\right)\right) \geqq M_{2}^{2} D\left(\omega\left(g^{\prime}{ }_{M_{2}}, z, G\right)\right) .
$$

Put $\delta=\frac{M_{2}}{M_{1}} \cdot$ Then

$M_{2}^{2} D\left(\omega\left(g^{\prime} M_{2}, z, G\right)\right)=M_{2}^{2} \times \frac{M_{1}}{M_{2}} D\left(\omega\left(g_{M_{1}}, z, G\right)\right)=M_{1} M_{2} D\left(\omega\left(g_{M_{1}}, z, G\right)\right)=$ $\frac{M_{2}}{M_{1}} \underset{c g_{M_{1}}}{D}\left(g_{M_{1}} U(z)\right)$. Hence by (3) $\left.\quad\left(\frac{1}{M_{2}}\right)_{c g_{M_{2}}}\left(g_{M_{2}} U(z)\right) \geqq\left(\frac{1}{M_{1}}\right)_{c g_{M_{1}}}{\left(g_{M_{1}}\right.}_{U} U(z)\right) \quad$ for $M_{2} \leqq M_{1}$ and $\left(\frac{1}{M}\right) \underset{c g_{M}}{D}\left(g_{M} U(z)\right) \quad$ increases as $\quad M \rightarrow 0 . \quad$ Put $\mathfrak{M}^{f}(U(z))=$ $\frac{1}{2 \pi} \lim _{M \rightarrow 0}\left(\frac{1}{M}\right) \underset{c g_{M}}{D}\left(g_{M} U(z)\right)$ and call $\mathfrak{M}^{f}(U(z))$ functiontheoretic mass of $U(z)$. Then we have the following

L.eMma 1. 1) Let $U_{1}(z)$ and $U_{2}(z)$ be two $F_{0} . S . H . s$ in $G$ and $U_{1}(z) \geqq U_{2}(z)$. Then

$$
\mathfrak{M l}^{f}\left(U_{1}(z)\right) \geqq \mathfrak{M C}^{f}\left(U_{2}(z)\right)
$$

2) Let $U_{m}(z)$ be F.S.H.s and $U_{m}(z) \uparrow U(z)$ as $m \rightarrow \infty$. Then

$$
\lim _{m=\infty} \mathfrak{M}^{f}\left(U_{m}(z)\right)=\mathfrak{M}^{f}(U(z))
$$

(1) is clear by $E\left[z: U_{1}(z)>M\right] \supset E\left[z: U_{2}(z)>M\right]$. At first we suppose $\mathfrak{M}^{f}(U(z))<\infty$. For any given $\varepsilon>0$, there exists a const. $M$ such that 
$\frac{1}{2 \pi M} \underset{c g_{M}}{D}\left(g_{M} U(z)\right)=\frac{1}{2 \pi} M D\left(\omega\left(g_{M}, z, G\right)\right) \geqq \mathfrak{M}^{f}(U(z))-\varepsilon . \quad$ Since $E\left[z: U_{m}(z)>M\right]=$ $g_{M, m} \uparrow g_{M}=E[z: U(z)>M] \quad$ as $\quad m \rightarrow \infty, \quad D\left(\omega\left(g_{M, m}, z, G\right)\right) \rightarrow D\left(\omega\left(g_{M}, z, G\right)\right)^{[4]} \quad$ as $m \rightarrow \infty$. Hence $\lim _{m=\infty} \mathfrak{M}^{f}\left(U_{m}(z)\right) \geqq \frac{1}{2 \pi} \lim _{m=\infty} M D\left(\omega\left(g_{M, m}, z, G\right)\right) \geqq \mathfrak{M}^{f}(U(z))-\varepsilon$. Let $\varepsilon \rightarrow 0$. Then $\lim _{m=\infty} \mathfrak{M}^{f}\left(U_{m}(z)\right) \geqq \mathfrak{M}^{f}(U(z))$.

Next by Lemma 1.1) $\lim _{m=\infty} \mathfrak{M l}^{f}\left(U_{m}(z)\right) \leqq \mathfrak{M l}^{f}(U(z)) . \quad$ If $\mathfrak{M}^{f}(U(z))=\infty$, we have similarly $\lim _{m=\infty} \mathfrak{M}^{f}\left(U_{m}(z)\right)=\infty$.

$\mathfrak{M}^{f}(U(z))$ of an F.S.H. $U(z)$ in $G$. For a compact domain $D$ in $G$, suppose that we can define functions $\left\{U_{n}(z)\right\}$ such that $U_{n}(z)$ is superharmonic in $G, U_{n}(z)$ is harmonic in $G-D, U_{n}(z) \geqq \min (M, U(z))$ on $D, U_{n}(z)=0$ on $\partial G$ except a set of cap. zero and $U_{n}(z)$ has M.D.I. over $G-D$. Let ${ }_{D}^{0} U^{M}(z)$ be the lower envelope of $\left\{U_{n}(z)\right\}$. Put ${ }_{D}^{0} U(z)=\lim _{M=\infty}{ }_{D}^{0} U^{M}(z)$ (clearly ${ }_{D}^{0} U(z) \leqq{ }_{D} U(z)$ ). Since $\partial D$ is compact, ${ }_{D}^{0} U(z)=0$ on $\partial G$ except a set of cap. zero. For non compact domain, ${ }_{D}^{0} U(z)$ is defined as ${ }_{D} U(z)$. For $U(z)$, put $\mathfrak{M}^{f}(U(z))=$ $\lim _{n=\infty} \mathfrak{M}^{f}\left(G_{n} U^{0}(z)\right)$, where $\left\{G_{n}\right\}$ is an exhaustion of $G$ with compact relative boundary.

$N$-Green's functions of $G$. Let $N_{n}(z, p)$ be a positive harmonic function in $(G-p) \cap R_{n}: p \in G$ such that $N_{n}(z, p)=0$ on $\partial G$ except a set of capacity zero, $N_{n}(z, p)$ has a logarithmic singularity at $p$ and $\frac{\partial}{\partial n} N_{n}(z, p)=0$ on $\partial R_{n} \cap G$. Then $N_{n}(z, p) \rightarrow N(z, p)$ in mean as $n \rightarrow \infty$ and $N(z, p)$ has M.D.I. (in this case the Dirichlet integral of $N(z, p)$ is taken with respect to $N(z, p)$ $+\log |z-p|$ in a neighbourhood of $p$ ). If $\partial G$ is composed of a finite number of analytic curves in $G$, we say that $\partial G$ is completely regular. Then as case that $\partial G$ is completely regular we see easily ${ }^{[5]}$

1). $N(z, p)=0$ on $\partial G$ except at most a set of cap. zero.

2). $D(\min (M, N(z, p)))=2 \pi M$.

3). For any domain $D \quad{ }_{D} N(z, p)=N(z, p)$ if $p \in D$ and ${ }_{D} N(z, p) \leqq N(z, p)$.

4). By 2) and 3) we have $\mathfrak{M}^{f}(N(z, p))=1$.

We show, for any point $z$ in $G$ and a positive const. $d$ there exists a const. $L(z, d)$ such that $N(z, p)<L(z, d)$ if $\operatorname{dist}(z, p)>d$.

Case 1. $\partial G$ has a continuum $\gamma$. Suppose $\gamma$ contains a small arc $C^{\prime}$ with endpoints $p_{1}$ and $p_{2}$. Let $C^{\prime \prime}$ be also an arc in $G$ connecting $p_{1}$ and $p_{2}$ so that $C^{\prime}+C^{\prime \prime}$ may enclose a simply connected domain $D$ of $R$. Let $C^{\prime \prime \prime}$ be a subarc in $C^{\prime \prime}$ such that $\operatorname{dist}\left(C^{\prime \prime \prime}, \partial G\right)>0$. Let $w(z)$ be a harmonic 
function in $D$ such that $w(z)=1$ on $C^{\prime \prime \prime}, w(z)=0$ on $\partial D-C^{\prime \prime \prime}$. Then $w(z)=0$ on $C^{\prime}$ and $\infty>\int_{C^{\prime}} \frac{\partial}{\partial n} w(z) d s>\delta>0$. Without loss of generality we can suppose $\operatorname{dist}(p, D)>d>$ diameter of $D$. Let $N^{*}(z, p)$ be an $N$-Green's function of $G+(C G \cap D)$. Then $N^{*}(z, p) \geqq N(z, p)$ and $N^{*}(z, p)$ is harmonic in a neighbourhood of $C^{\prime \prime \prime}$. Hence by Harnack's theorem, there exists a const. $K$ such that $\max _{z \in C^{\prime \prime \prime}} N^{*}(z, p) \leqq K \min _{z \in C^{\prime \prime \prime}} N^{*}(z, p)$. Let $L=\max _{z \in C^{\prime \prime \prime}} N^{*}(z, p)$. Then $N^{*}(z, p) \geqq \frac{L}{K} w(z)$ in $D$ and $2 \pi>\int_{C^{\prime}} \frac{\partial}{\partial n} N^{*}(z, p) d s \geqq \frac{L \delta}{K}$, whence $L \leqq \frac{2 \pi K}{\delta}$. Hence also by Harnack's theorem, for any point $z$, there exists a const. $L(z, d)$ such that $N(z, p) \leqq L(z, d)$ if $\operatorname{dist}(z, p) \geqq d$. If $G$ has a continuum $\gamma$ (is not an analytic curve). Map $D$ onto $|\xi|<1$. Then the image of $\gamma$ is an analytic curve. Hence even when $\gamma$ is not analytic curve, we have the same conclusion.

Case 2. $\partial G$ has no continuum. By $N(z, p) \neq \infty$, we can find a point $z_{0}$ in $\partial G$ such that $\inf _{z \rightarrow z_{0}} N(z, p)=0$. Let $D$ be a simply connected domain in $R$ such that $\partial D$ is an analytic curve, $D \ni z_{0}$ and $(\partial D \cap \partial G)=0$. Then $\operatorname{dist}(\partial D, \partial G)>0$. We suppose $p \notin D$, dist $(p, D)>\operatorname{diameter}$ of $D$ and $\operatorname{dist}\left(\partial G_{n}, \partial D\right)>0$, where $\left\{G_{n}\right\}$ is an exhaustion of $G$. Let $U_{m, n}(z)$ be a harmonic function in $G_{n} \cap R_{m}$ such that $U_{m, n}(z)=0$ on $\partial G_{n} \cap R_{m}, \frac{\partial}{\partial n} U_{m, n}(z)=0$ on $\partial R_{m} \cap G_{n}$ and $U_{m, n}(z)$ has a logarithmic singularity at $p: R_{m} \supset D$. Then $\lim _{m} \lim _{n} U_{m, n}(z)=N(z, p)$. Let $w_{n}(z)$ be a harmonic function in $G_{n} \cap D$ such that $w_{n}(z)=0$ on $\partial G_{n} \cap D,=1$ on $\partial D$. Then $\frac{U_{m, n}(z)}{L_{m, n}} \leqq w_{n}(z)$ and $\frac{2 \pi}{L_{m, n}} \geqq \int_{\partial G_{n}} \frac{1}{L_{m, n}} \frac{\partial}{\partial n} U_{m, n}(z) d s \geqq \int_{\partial G_{n}} w_{n}(z) d s>0$, where $L_{m, n}=\min _{z \in \partial D} U_{m, n}(z)$. Now by $\int_{\partial D} \frac{\partial}{\partial n} U_{m, n}(z) d s=\int_{\partial G_{n} \cap D} \frac{\partial}{\partial n} U_{m, n}(z) d s>0$ and $\int_{\partial D} \frac{\partial}{\partial n} w_{n}(z) d s=$ $\int_{\partial G_{n} \cap D} \frac{\partial}{\partial n} w_{n}(z) d s>0$ we have $\int_{\partial D} \frac{\partial}{\partial n} U_{m, n}(z) d s \geqq L_{m, n} \int_{\partial D} \frac{\partial}{\partial n} w_{n}(z) d s$. Let $n \rightarrow \infty$ and $m \rightarrow \infty$. Then since $\partial D$ is compact

$$
2 \pi \geqq \int_{\partial D} \frac{\partial}{\partial n} N(z, p) d s \geqq L \int_{\partial D} \frac{\partial}{\partial n} w(z) d s=L \delta>0,
$$

where $L=\min _{z \in \partial D} N(z, p)$ and $\int_{\partial D} \frac{\partial}{\partial n} w(z) d s=\delta$. 
$\inf _{z \rightarrow z_{0}} N(z, p)=0$ implies $w(z) \neq 1$ and $\delta>0$. Hence $L \leqq \frac{2 \pi}{\delta}$. Whence by Harnack's theorem we have the same conclusion.

Let $\left\{p_{i}\right\}$ in $G$ be a divergent sequence tending to the boundary of $R$ or $\partial G$. Then $N\left(z, p_{i}\right) \leqq L(z, d)<\infty$ for any point $z$ if $\operatorname{dist}\left(z, p_{i}\right) \geqq d$. Then we can choose a subsequence $\left\{p_{i^{\prime}}\right\}$ such that $N\left(z, p_{i^{\prime}}\right)$ converges uniformly to a harmonic function denoted by $N(z, p)$ and we call $\left\{p_{i^{\prime}}\right\}$ a fundamental sequence determining an ideal point $p$. We denote by $B$ the all the ideal points $(p$ may be on $\partial G)$. We show $N(z, p)=0(p \in B)$ for a regular boundary point $z$ of $\partial G$.

Case 1. $\partial G$ has a continuum $r$ with endpoints $q_{1}$ and $q_{2}$. Let $z_{0} \in \gamma, z_{0} \neq q_{1}$ and $\neq q_{2}$. Let $C$ be an analytic curve in $G$ connecting $q_{1}$ and $q_{2}$ so that $C+r$ may enclose a simply connected domain $D$ in $R$ and $D \oplus p_{i} \quad(i=1,2$, ... ), where $\left\{p_{i}\right\}$ is a fundamental sequence determining $p$. Map $D$ conformally onto $|\xi|<1$. Then $\gamma$ and $C$ are mapped onto the images denoted by the same notations for simplicity. $N\left(z, p_{i}\right)=0$ on $\gamma+(\partial G \cap D)$ except a set of cap.zero. Let $N^{*}\left(z, p_{i}\right)$ be an $N$-Green's function of $G+(C G \cap D)$. Then there exists a const. $L^{*}\left(t_{0}\right)$ such that $\infty>L^{*}\left(t_{0}\right) \geqq$ $N^{*}\left(t_{0}, p_{i}\right)=\frac{1}{2 \pi} \int_{C} N^{*}\left(\xi, p_{i}\right) \frac{\partial}{\partial n} G\left(\xi, t_{0}\right) d s$ for any $i$, where $G(\xi, t)$ is the Green's function of $D$. On the other hand, there exists a const. $M$ such that $0<M<\frac{\partial}{\partial n} G\left(\xi, t_{0}\right)$ on $C$, whence $\int_{C} N^{*}\left(\xi, p_{i}\right) d s \leqq \frac{2 \pi L^{*}\left(t_{0}\right)}{M}$. Let $U(\xi)$ be a harmonic function in $|\xi|<1$ such that $U(\xi)=N^{*}\left(\xi, p_{i}\right)$ on $|\xi|=1$. Then $N^{*}\left(t, p_{i}\right)=U(t)=\frac{1}{2 \pi} \int_{C} N^{*}\left(\xi, p_{i}\right) \frac{1-r^{2}}{1-2 r \cos (\theta-\varphi)+r^{2}} d \varphi: t=r e^{i \theta}$. Since $\xi_{0}=\xi\left(z_{0}\right) \in \gamma$, there exists a neighbourhood $v\left(\xi_{0}\right)$ such that $v\left(\xi_{0}\right) \cap C=0$. Now there exists a const. $M^{\prime}$ such that $1-2 r \cos (\theta-\varphi)+r^{2} \geqq M^{\prime}$ for $e^{i \varphi} \notin \gamma$ and $\xi \in v\left(\xi_{0}\right): \xi=r e^{i \theta}$. Hence $U(\xi) \leqq \frac{2 \pi L^{*}\left(t_{0}\right)}{M M^{\prime}}\left(1-r^{2}\right)$ for $\xi \in v\left(\xi_{0}\right)$. Then by Fatou's lemma $N^{*}(\xi, p) \leqq \frac{2 \pi L^{*}\left(t_{0}\right)}{M M^{\prime}}\left(1-r^{2}\right)$ and by $N\left(z, p_{i}\right) \leqq N^{*}\left(z, p_{i}\right)$ we have $N(z, p) \rightarrow 0$ as $z \rightarrow z_{0}$.

Case 1.2. $z_{0} \in$ endpoint of an arc $\gamma$. Let $D$ be a domain such that $\partial D+\gamma$ encloses a simply connected domain $D-\gamma$. Map $D-\gamma$ onto $|\xi|<1$. Then the image $\left(z_{0}\right)$ of $z_{0}$ is an inner point of the image of $r$. Then as case 1.1. we have $N(z, p) \rightarrow 0$ as $z \rightarrow z_{0}$.

Case 2. $z_{0}$ is a regular point and $z_{0}$ is not contained in any continuum. Let 
$D$ be a simply connected domain such that $D \ni z_{0}$ and $\partial D \cap \partial G=0$ and $D \oplus p_{i}(i=1,2, \ldots)$. Then since $(\partial D \cap \partial G)=0$ implies $\operatorname{dist}(\partial D, \partial G)>0$, there exist const.s $L_{1}$ and $L_{2}$ such that $N\left(z, p_{i}\right) \leqq L_{1}$ and $N\left(z, p_{i}\right) \geqq L_{2}$ on $\partial D$. Whence there exists a const. $M$ such that $N\left(z, p_{\imath}\right) \leqq M N\left(z, p_{1}\right)$ in $D$. Hence $\lim _{z \rightarrow z_{0}} N(z, p) \leqq M \lim _{z \rightarrow z_{0}} N\left(z, p_{1}\right)=0$. Thus $N(z, p)=0$ on $\partial G$ except at most a set of capacity zero. $D(\min (M, N(z, p))) \leqq \lim _{i} D\left(\min \left(M, N\left(z, p_{i}\right)\right)\right) \leqq 2 \pi M$. Hence we can define ${ }_{D} N(z, p)$ for any compact domain. $N\left(z, p_{i}\right) \rightarrow N(z, p)$ uniformly on $\partial D$ as $i \rightarrow \infty$. Hence by ${ }_{D} N\left(z, p_{i}\right) \leqq N\left(z, p_{i}\right)$ we have ${ }_{D} N(z, p) \leqq N(z, p)$. Next we have at once $\mathfrak{M i}^{f}(N(z, p)) \leqq \frac{D(\min (M, N(z, p)))}{2 \pi M} \leqq 1$. Hence we have the following.

Lemma 2. $N(z, p)$ is an $F_{0} . S . H$. in $G$ such that $D(\min (M, N(z, p))) \leqq 2 \pi M$ and $\mathfrak{M}^{f}(N(z, p)) \leqq 1$ for $p \in G+B$.

$N$-Martin topology in $G$. Let $D$ be a compact disc in $G$ and $p_{0}$ be a fixed point in $D$. we define the distance between two points $p_{1}$ and $p_{2}$ of $G+B$ as

$$
\delta\left(p_{1}, p_{2}\right)=\sup _{z \in D}\left|\frac{N\left(z, p_{1}\right)}{1+N\left(z, p_{1}\right)}-\frac{N\left(z, p_{2}\right)}{1+N\left(z, p_{2}\right)}\right| .
$$

Then the topology induced by this metric is homeomorphic to the original topology in $G$. In the following we use this topology. $\delta\left(p, p_{i}\right) \rightarrow 0$ if and only if $N\left(z, p_{i}\right) \rightarrow N(z, p)$. Put $G_{\delta}=E\left[z: N\left(z, p_{0}\right)>\delta\right]$. Then the distance between $G_{\delta}$ and $C G_{\delta^{\prime}}=E\left[z: N(z, p) \leqq \delta^{\prime}\right]$ is not less than $\frac{\delta-\delta^{\prime}}{4}$, if $0<\delta^{\prime}<\delta<1$. In fact, by the symmetry of $N(p, q)$ we have at once

$$
\delta\left(q_{1}, q_{2}\right) \geqq\left|\frac{N\left(p_{0}, q_{1}\right)}{1+N\left(p_{0}, q_{1}\right)}-\frac{N\left(p_{0}, q_{2}\right)}{1+N\left(p_{0}, q_{2}\right)}\right| \geqq \frac{\delta-\delta^{\prime}}{4}: q_{1} \in G_{\delta} \text { and } q_{2} \in C G_{\delta^{\prime}} .
$$

Also we easily see $B \cap \bar{G}_{\delta}$ is compact for every $\delta>0$.

Potentials. Let $\mu>0$ be a positive mass distribution on $G+B$ such that $\int d \mu(p)<\infty$ and put $U(z)=\int N(z, p) d \mu(p)$. If a potential $U(z)=0$ on $\partial G$ except at most a set of cap. zero, we call $U(z)$ a regular potential. Then we have the following

TheOREM 1.1). $\quad D(\min (M, U(z))) \leqq 2 \pi M \int d \mu$.

2). Let $D$ be a compact or non compact domain. Then ${ }_{D} U(z)=\int{ }_{D} N(z, p) d \mu(p)$.

3). Let $\mu_{\varepsilon}$ be the restriction of $\mu$ on $G_{\varepsilon}=E\left[z: N\left(z, p_{0}\right)>\varepsilon\right]$. Then

$$
U(z)=\lim _{\varepsilon \rightarrow 0} \int N(z, p) d \mu_{\varepsilon}(p) .
$$


4). If $U(z)$ is a regular potential, $U(z)$ is an F.S.H. in $G$ with $\mathfrak{M}^{f}(U(z)) \leqq \int d \mu$.

Proof of 1). For any number $\varepsilon>0$ we can find a compact set $K$ in $H=E[z: U(z) \leqq M]$ such that $D(\min (M, U(z)))<D_{K}(U(z))+\varepsilon$. Since $N(z, p)$ is a continuous function of $p$ for fixed $z, U(z)$ can be approximated on $K$ by a sequence of linear forms : $U_{i}(z)=\sum_{j=1}^{j(i)} \lambda_{i j} N\left(z, p_{j}\right), \lambda_{i j} \geqq 0, \quad p_{j} \in G$, $\int d \mu=\sum_{j} \lambda_{i j}: i=1,2, \ldots$ Hence $\underset{K}{D}(U(z)) \leqq \lim _{i} \underset{K}{D}\left(U_{i}(z)\right)$. Also $U_{i, n}(z) \rightarrow U_{i}(z)$ in mean as $n \rightarrow \infty$, where $U_{i, n}(z)=\sum \lambda_{i j} N_{n}\left(z, p_{j}\right)$ and $N_{n}\left(z, p_{j}\right)$ is a harmonic function in $G \cap R_{n}$ such that $N_{n}\left(z, p_{j}\right)=0$ on $\partial G \cap R_{n}$ except a set of cap. zero, $\frac{\partial}{\partial n} N_{n}\left(z, p_{j}\right)=0$ on $\partial R_{n} \cap G$ and $N_{n}\left(z, p_{j}\right)$ has logarithmic singularity at $p_{j}$. Put $\underset{\varepsilon, i, n}{H}=E\left[z: U_{i, n}(z)<M+\varepsilon\right]$. Then $\underset{\varepsilon, i, n}{H} \supset K$ for $n \geqq n_{0}$, where $n_{0}$ is a sufficiently large number. We can prove (with some modefication to the fact $N_{n}\left(z, p_{j}\right)=0$ on $\partial G$ except a set of cap. zero instead of $N_{n}\left(z, p_{j}\right)=0$ on $\partial G)$ that $\underset{H_{\varepsilon, i, n}}{D}\left(U_{i, n}(z)\right)=2 \pi(M+\varepsilon) \int d \mu(p)$. Let $n \rightarrow \infty, \quad i \rightarrow \infty$ and then $\varepsilon \rightarrow 0$. Then $D\left(\min (M, U(z)) \leqq 2 \pi M \int d \mu(p)\right.$.

Proof of 2). Put $D_{n}=D \cap G_{n}$. Then $D_{n}$ is compact. Put $N^{M}(z, p)=$ $\min (M, N(z, p))$. Then $N^{M}(z, p)$ is uniformly continuous with respect to $p$ on $D_{n}$. Hence $\int N^{M}(z, p) d \mu(p)$ can be approximated uniformly on $D_{n}$ by a sequence of linear forms: $\quad U_{i}(z)=\sum_{j=1}^{j(i)} \lambda_{i, j} N^{M}\left(z, p_{j}\right), \lambda_{i, j} \geqq 0$. Clearly $\quad D_{n} U_{i}(z)=\sum \lambda_{i j} D_{n} N^{M}\left(z, p_{j}\right)$. Let $i \rightarrow \infty$. Then ${ }_{D_{n}}\left(\int N^{M}(z, p) d \mu(p)\right)=$ $\int D_{n} N^{M}(z, p) d \mu(p)$. Now by $\int N^{M}(z, p) d \mu(p) \uparrow U(z)$ as $M \rightarrow \infty$, we have ${ }_{D_{n}}\left(\int N^{M}(z, p) d \mu(p)\right) \uparrow \quad{ }_{D_{n}}\left(\int N(z, p) d \mu(p)\right)=D_{n} U(z)$ and $\int{ }_{D_{n}} N^{M}(z, p) d \mu(p) \uparrow$ $\int D_{n} N(z, p) d \mu(p)$ as $M \uparrow \infty$. Hence $D_{n} U(z)=\int D_{n} N(z, p) d \mu(p)$. Since $D_{n} N(z, p)$ $\uparrow{ }_{D} N(z, p)$ and $D_{n} U(z) \uparrow D U(z)$ as $n \rightarrow \infty,{ }_{D}\left(\int N(z, p) d \mu(p)\right)=\lim _{n}\left({ }_{D_{n}} \int N(z, p)\right.$ $d \mu(p))=\int \lim _{n} D_{n} N(z, p) d \mu(p)=\int D N(z, p) d \mu(p)$.

Proof of 3). Suppose $p \notin G_{\varepsilon}$. Let $\left\{p_{i}\right\}$ be a fundamental sequence determining $p \in B$ (if $p \in G$, put $p_{i}=p$ ). Then $\delta\left(p, p_{i}\right) \rightarrow 0$ as $i \rightarrow \infty$. Then by $\operatorname{dist}\left(C G_{\varepsilon}, G_{2 \varepsilon}\right) \geqq \frac{\varepsilon}{4}$, (if $2 \varepsilon<1$ ), $p_{i} \notin G_{2 \varepsilon}$ for $i \geqq i_{0}$, where $i_{0}$ is a 
number. Hence $N\left(p_{0}, p_{i}\right) \leqq 2 \varepsilon$ and $N\left(p_{0}, p\right) \leqq 2 \varepsilon$ for $p \in(B+G)-G_{2 \varepsilon}$. Let $\mu_{\varepsilon}^{\prime}=\mu-\mu_{\varepsilon}$. Then ${ }_{\varepsilon} U^{\prime}\left(p_{0}\right)=\int N\left(p_{0}, p\right) d \mu_{\varepsilon}^{\prime}(p) \leqq 2 \varepsilon \int d \mu^{\prime} . \quad U_{\varepsilon}^{\prime}(z)$ is harmonic in $G-G_{2 \varepsilon}$. Hence by Harnack's theorem $U_{\varepsilon}^{\prime}(z) \rightarrow 0$ as $\varepsilon \rightarrow 0$ at every point z. Hence we have 3 ).

Proof of 4). $\underset{c g_{M}}{D}\left(g_{M} U(z)\right) \leqq D(\min (M, U(z))) \leqq 2 \pi M \int d \mu$ by (1). Hence by definition $\mathfrak{M}^{f}(U(z))=\lim _{M=0} \frac{\left.\underset{c g_{M I}}{D\left(g_{M}\right.} U(z)\right)}{2 \pi M} \leqq \int d \mu$, where $g_{M}=E[z: U(z)>M]$. By (2) ${ }_{D} U(z) \leqq U(z)$, hence $U(z)$ is an $\mathrm{F}_{0} . \mathrm{S} . \mathrm{H}$. in $G$ with $\mathfrak{M}^{f}(U(z)) \leqq \int d \mu$.

THeOREm 2. Let $U(z)$ be an $F_{0}$. S.H. in $G$ with $\mathfrak{M}^{f}(U(z))<\infty$. Then $U(z)$ can be represented by a positive mass distribution $\mu$ on $G+B$ such that $\int d \mu \leqq \mathfrak{M}^{f}(U(z))$.

Let $D$ and $D^{\prime}$ be compact domains in $G$ with finite number of analytic curves as their relative boundaries such that dist $\left(D, \partial D^{\prime}\right)>0$ and $D^{\prime} \supset D$. Let $M$ be a number. Put $U^{M}(z)=\min (M, U(z))$. Then $U^{M}(z)$ is also an F. S.H. in $G$ and $\mathfrak{M}^{f}\left(U^{M}(z)\right) \leqq \mathfrak{M}^{f}(U(z))$. Let $\delta$ be a positive const. such that $\delta<\min _{z \in \bar{D}^{\prime}} U^{M}(z)$. Then $\partial H_{\delta} \cap \bar{D}^{\prime}=0$, where $H_{\alpha}=E\left[z:{ }_{D} U^{M}(z)>\alpha\right]$. Then ${ }_{D} U^{M}(z)=\delta \omega\left(H_{\delta}, z, G\right)$ in $C H_{\delta}$. Hence we can find a const. $\delta^{\prime}$ such that $\delta^{\prime}<\delta$ and $\partial H_{\delta^{\prime}}$ is a regular niveau of $\omega\left(H_{\delta}, z, G\right)$. Hence $\mathfrak{M}^{f}\left(U^{M}(z)\right) \geqq \mathfrak{M}^{f}\left({ }_{D} U^{M}(z)\right)=\lim _{M^{\prime} \rightarrow 0} \frac{D\left(\min \left(M^{\prime},{ }_{D} U^{M}(z)\right)\right)}{2 \pi M^{\prime}}=\frac{D\left(\min \left(\delta,{ }_{D} U^{M}(z)\right)\right)}{2 \pi \delta}=$ $\frac{1}{2 \pi} \int_{\partial H_{\delta^{\prime}}} \frac{\partial}{\partial n}{ }_{D} U^{M}(z) d s$

Put

$$
U\left(\delta^{\prime}, z\right)={ }_{D} U^{M}(z)-\delta^{\prime}+\delta^{\prime} \omega\left(D, z, H_{\delta^{\prime}}\right) \text { in } H_{\delta^{\prime}}-D .
$$

Then $U\left(\delta^{\prime}, z\right)$ is a harmonic function in $H_{\delta^{\prime}}-D$ such that $U\left(\delta^{\prime}, z\right)={ }_{D} U^{M}(z)$ on $\partial D,={ }_{D} U^{M}(z)-\delta^{\prime}=0$ on $\partial H_{\delta^{\prime}}$ except a set of cap. zero and $U\left(\delta^{\prime}, z\right)$ has M.D.I. over $H_{\delta^{\prime}}-D$, because both ${ }_{D} U^{M}(z)$ and $\omega\left(D, z, H_{\delta^{\prime}}\right)$ have M.D.I.s over $H_{\delta^{\prime}}-D$. Now by the regularity of $\partial H_{\delta^{\prime}}$

$$
0 \leqq \int_{\partial H_{\delta^{\prime}}} \frac{\partial}{\partial n} \omega\left(D, z, H_{\delta^{\prime}}\right) d s=-\int_{\partial D} \frac{\partial}{\partial n} \omega\left(D, z, H_{\delta^{\prime}}\right) d s=a\left(\delta^{\prime}\right) .
$$

Since $\int_{\partial H_{\delta^{\prime}}} \frac{\partial}{\partial n} \omega\left(D, z, H_{\delta^{\prime}}\right) d s \downarrow$ as $\delta^{\prime} \downarrow$ 


$$
\delta^{\prime} \int_{\partial H_{\delta^{\prime}}} \frac{\partial}{\partial n} \omega(D, z, H) d s \downarrow 0 \text { as } \delta^{\prime} \rightarrow 0 .
$$

Hence $2 \pi \mathfrak{M}^{f}\left({ }_{D} U^{M}(z)\right)=\lim _{\delta^{\prime} \rightarrow 0} \int_{\partial H_{\delta^{\prime}}} \frac{\partial}{\partial n} D^{M}(z) d s=\lim _{\delta^{\prime} \rightarrow 0} \int_{\partial H_{\delta^{\prime}}} \frac{\partial}{\partial n}\left(U\left(\delta^{\prime}, z\right)\right.$

$$
\left.-\delta^{\prime} \omega\left(D, z, H_{\delta^{\prime}}\right)\right) d s=\lim _{\delta^{\prime} \rightarrow 0} \int_{\partial H_{\delta}} \frac{\partial}{\partial n} U\left(\delta^{\prime}, z\right) d s .
$$

Hence for any $\varepsilon>0$ we can find a const. $\delta^{*}$ such that $\partial H_{\delta^{*}}$ is regular and

$$
2 \pi \mathfrak{M}^{f}\left({ }_{D} U^{M}(z)\right) \geqq \int_{\partial H_{\delta} *}-\frac{\partial}{\partial n} U\left(\delta^{*}, z\right) d s-\varepsilon .
$$

Since ${ }_{D} U^{M}(z)$ is an $\mathrm{F}_{0} . \mathrm{S} . \mathrm{H}$. in $G$, there exists a uniquely determined positive mass distribution $\mu$ on $\bar{D}$ such that

$$
{ }_{D} U^{M}(z)=\int N(z, p) d \mu(p) .
$$

Let $N^{\prime}(z, p)$ be an $N$-Green's function of $H_{\delta^{*}}+D$ with pole at $p$. Then $N_{n}^{\prime}(z, p)$ is uniformly bounded on $\partial D^{\prime}$ for $p \in D^{\prime \prime}$ and $N_{n}^{\prime}(z, p) \rightarrow N^{\prime}(z, p)$ in mean as $n \rightarrow \infty$, where $D^{\prime \prime}$ is another domain such that $D \subset D^{\prime \prime} \subset D^{\prime}$ and $\operatorname{dist}\left(\partial D, \partial D^{\prime \prime}\right)>0$ and $\operatorname{dist}\left(\partial D^{\prime \prime}, \partial D^{\prime}\right)>0$ and $N_{n}^{\prime}(z, p)$ is a harmonic function in $\left(\left(H_{\delta^{*}}+D\right) \cap R_{n}\right)-p$ such that $N_{n}^{\prime}(z, p)$ has a logarithmic singularity at $p$, $N_{n}^{\prime}(z, p)=0$ on $\partial H_{\delta^{*}}$ and $\frac{\partial}{\partial n} N_{n}^{\prime}(z, p)=0$ on $\partial R_{n} \cap\left(H_{\delta^{*}}+D\right)$. Then by the regularity of $\partial H_{\delta^{*}}{ }^{[5]}$

$$
\int_{\partial H_{\delta^{*}}} \frac{\partial}{\partial n} N^{\prime}(z, p) d s=\lim _{n=\infty} \int_{\partial H_{\delta^{*}} \cap R_{n}} \frac{\partial}{\partial n} N_{n}^{\prime}(z, p) d s=2 \pi .
$$

$N^{\prime}$-Martin topology induced by $N^{\prime}(z, p)$ is homeomorphic to $N$-Martin topology on $G+B$ in $\left(D+H_{\delta^{*}}\right) \cap G$. Hence $\mu$ can be approximated by a sequence of points masses : $\sum_{j=1}^{i(j)} \lambda_{i j}\left(p_{i j}\right)$ uniformly in $D$, i.e. both $\int N(z, p) d \mu(p)$ and $\int N^{\prime}(z, p) d \mu(p)$ can be approximated by sequences of linear forms $U_{i}(z)=\sum_{j=1}^{j(i)} \lambda_{i j} N\left(z, p_{i j}\right) \quad$ and $\quad U_{i}^{\prime}(z)=\sum_{j=1}^{j(i)} \lambda_{i j}^{\prime} N^{\prime}\left(z, p_{i j}\right)$, where $\lambda_{i j}=\lambda_{i j}^{\prime} \geqq 0 \quad$ and $p_{i j} \in D^{\prime \prime}$. Hence $\int N(z, p) d \mu(p)-\int N^{\prime}(z, p) d \mu(p)$ is full harmonic in $H_{\delta^{*}}+D$ and $=\delta^{*}$ on $\partial H_{\delta^{*}}$. Hence by the maximum principle $\int N(z, p) d \mu(p)-$ $\int N^{\prime}(z, p) d \mu(p) \equiv \delta^{*}$ in $H_{\delta^{*}}+D$ 
Now $\omega\left(D, z, H_{\delta^{*}}\right)$ is represented by a positive mass distribution $\mu^{*}$ on $\bar{D}$. Hence by (4) $U\left(\delta^{*}, z\right)=\int N^{\prime}(z, p) d\left(\mu+\delta^{*} \mu^{*}\right)(p)$ in $H_{\delta^{*}}+D$. Whence by (7) $\int_{\partial H_{\delta^{*}}} \frac{\partial}{\partial n} U\left(\delta^{*}, z\right) d s=\int_{\partial H_{\delta^{*}}} \frac{\partial}{\partial n} \int N^{\prime}(z, p) d\left(\mu+\delta^{*} \mu^{*}\right)(p) d s=\iint_{\partial H_{\delta^{*}}} \frac{\partial}{\partial n} N^{\prime}(z, p) d s$ $d\left(\mu+\delta^{*} \mu^{*}\right)(p)=2 \pi \int d\left(\mu+\delta^{*} \mu^{*}\right) \geqq 2 \pi \int d \mu$.

Hence by (6) $2 \pi^{\mathfrak{M}} \mathfrak{M}^{f}\left({ }_{D} U^{M}(z)\right) \geqq 2 \pi \int d \mu-\varepsilon$.

Let $\varepsilon \rightarrow 0$. Then $\mathfrak{M}^{f}(U(z)) \geqq \mathfrak{M}^{f}\left({ }_{D} U(z)\right) \geqq \mathfrak{M}^{f}\left({ }_{D} U^{M}(z)\right) \geqq \int d \mu(p)$ and ${ }_{D} U^{M}(z)$ is representable by a mass distribution $\mu$ on $\bar{D}$ of total mass $\leqq \mathfrak{M}^{f}(U(z))$ for every $M$. Let $\left\{G_{n}\right\}$ be an exhaustion of $G$. Then $G_{n} U^{M}(z)$ is representable by mass distribution $\mu_{n}^{M}$ on $\bar{G}_{n}$. Then $\left\{\mu_{n}^{M}\right\}$ has a weak limit $\mu_{n}$ on $\bar{G}_{n}$ as $M \uparrow \infty$. Also $\left\{\mu_{n}\right\}$ has a weak limit $\mu$ on $G+B$ such that $U(z)=$ $\int N(z, p) d \mu(p)$ and $\int d \mu(p) \leqq \mathfrak{M}^{f}(U(z))$ as $n \rightarrow \infty$. Thus we have the theorem.

Corollary. Let $\mu$ be a positive mass distribution on a compact set $F$ in $G$. Then $U(z)=\int N(z, p) d \mu(p)$ is an $F_{0}$.S.H. in $G$ with $\mathfrak{M}^{f}(U(z))=\int d \mu$.

Let $D_{1} \supset D_{2}$ be two domains in $G$ such that $D_{1} \supset D_{2} \supset F$, dist $\left(D_{2}, \partial D_{1}\right)>0$ and $\operatorname{dist}\left(F, \partial D_{2}\right)>0$. Then $N(z, p): p \in F$ is uniformly bounded on $\partial D_{1}$. Put $L=\max _{p \in F}\left(\max _{z \in \partial D_{1}} N(z, p)\right)$ and $L^{\prime}=\min _{z \in \partial D_{1}} N\left(z, p_{0}\right)$, where $p_{0}$ is a fixed point in $F$. Then $N\left(z, p_{0}\right) \geqq \frac{L^{\prime}}{L} N(z, p)$ in $G-D_{2}$ for any $p \in F$. Hence $U(z)=0$ on $\partial G$ except at most a set of cap. zero. Also ${ }_{D_{1}} U(z)=U(z)$ and $\mathfrak{M}^{f}(U(z))=\mathfrak{M}^{f}\left({ }_{D_{1}} U(z)\right)$. By Theorem $2 U(z)$ is representable by a mass distribution $\mu^{*}$ on $\bar{D}_{1}$ such that $\mathfrak{M}^{f}(U(z)) \geqq \int d \mu^{*}$. But since $D_{1}$ is compact, by the uniqueness of distribution, $\mu=\mu^{*}$ and $\operatorname{MM}^{f}(U(z)) \geqq \int d \mu$. On the other hand, by Theorem 1.2. $\mathfrak{M}^{f}(U(z)) \leqq \int d \mu$. Hence $\mathfrak{M}^{f}(U(z))=\int d \mu$ and $U(z)$ is an $\mathrm{F}_{0}$. S.H. in $G$.

Theorem 3. Let $U(z)$ be an F.S.H. in $G$ with $\mathfrak{M I}^{f}(U(z))<\infty$. Then $U(z)$ is representable by a positive mass distribution $\mu$ with $\int d \mu \leqq \mathfrak{M}^{f}(U(z))$. Conversely a potential $U(z)=\int N(z, p) d \mu(p)$ is an F.S.H. in $G$ with $\mathfrak{M}^{f}(U(z)) \leqq \int d \mu$.

Let $\left\{G_{n}\right\}$ be an exhaustion of $G$. Suppose $U(z)$ is an F.S.H. in $G$. Then $G_{n} U(z)$ can be defined and there exists a mass distribution $\mu_{n}$ on $\bar{G}_{n}$ such that $G_{n} U(z)=\int N(z, p) d \mu_{n}(p)$ and $\int d \mu_{n} \leqq \mathfrak{M}^{f}\left(G_{n} U(z)\right) \leqq \mathfrak{M}^{f}(U(z))$. Hence $\left\{\mu_{n}\right\}$ has a weak limit $\mu$ such that $U(z)=\lim _{n} G_{n} U(z)=\int N(z, p) d \mu(p)$. Let $U(z)$ be a potential. Let $D$ be a compact domain. Then ${ }_{D} U(z)=$ 
$\int{ }_{D} N(z, p) d \mu(p)$ and ${ }_{D} U(z)$ can be defined and ${ }_{D} U(z) \leqq U(z)$. Now $N(z, p)$ is an $\mathrm{F}_{0} . \mathrm{S}$.H. in $G$ with $\mathfrak{M l}^{f}(N(z, p)) \leqq 1$ by Theorem 1 , whence by the corollary ${ }_{D} N(z, p)=\int_{D} N(z, q) d \mu_{p}(q): \int d \mu_{p}(q) \leqq 1$. Hence ${ }_{D} U(z)=\iint N(z, q) d \mu_{p}(q) d \mu(p)=$ $\int N(z, q) d \mu(q): \mu(q)=\int \mu_{p}(q) d \mu(p)$ and $\mathfrak{M M}^{f}\left({ }_{D} U(z)\right)=\int d \mu(q) \leqq \int d \mu$ for any $D$. Hence $U(z)=\lim _{n} G_{n} U(z)$ is an F.S.H. in $G$ with $\mathfrak{M}^{f}(U(z)) \leqq \int d \mu$.

Remark. Let $U(z)$ be an F.S.H. in $G$ with $\mathfrak{M}^{f}(U(z))<\infty$. Then $V_{M}=E[z: U(z)>M]$ is so thinly distributed in a neighbourhood of $\partial G$. In fact, $D\left(\omega\left(V_{M}, z, G\right)\right) \leqq 2 \pi M M^{f}(U(z))$. This means $V_{M}$ is thin. If $V_{M}$ is very thik, $D\left(\omega\left(V_{M} \cap G_{n}, z, G\right)\right) \uparrow \infty$ as $n \rightarrow \infty$.

Let $D$ be a domain. Then by Theorems 1 and 2 we can consider the mass distribution of ${ }_{v_{n}(p)} N(z, p)$, where $v_{n}(p)=E\left[z \in G+B: \operatorname{dist}(z, p)<\frac{1}{n}\right]$. As case that $\partial G$ is completely regular we have the following ${ }^{[6]}$

Lemma 3. Let $U(z)$ be an F.S.H. (or F.S.H.) in $G$ with $\mathfrak{M}^{f}(U(z))<\infty$. Let $F$ be a closed set. We define ${ }_{F} U(z)$ by $\lim _{n=\infty} F_{n} U(z)$, where $F_{n}=E[z \in G+B$ : $\left.\operatorname{dist}(z, F) \leqq \frac{1}{n}\right]$. Then

1). ${ }_{F}\left({ }_{F} U(z)\right)={ }_{F} U(z)$, if $\omega(F, z, G)=0$.

2). $\quad \omega(F, z, G)={ }_{F} \omega(F, z, G)$, if $\omega(F, z, G)>0$.

$\mathfrak{M l}^{f}(N(z, p)) \leqq 1$ for $p \in G+B$. If $\partial G$ is completely regular $\mathfrak{M}^{f}(N(z, p))$ $=\frac{1}{2 \pi} \int_{\partial G} \frac{\partial}{\partial n} N(z, p) d s=1$.

But in the present case $\mathfrak{M}^{f}(N(z, p))$ is not necessarily equal to 1 . Then we shall prove the following

Theorem 4. 1). Put $\mathfrak{M}(p)=\mathfrak{M}^{f}(N(z, p))$. Then $\mathfrak{M}(p)=1$ for $p \in G$ and $\mathfrak{M}(p)$ is lower semicontinuous.

2). Put $\phi\left(v_{n}(p)\right)=\mathfrak{M}^{f}\left({ }_{v_{n}(p)} N(z, p)\right)$. Then $\phi\left(v_{n}(p)\right)=1$ for $p \in G$ and $\phi\left(v_{n}(p)\right)$ is lower semicontinuous. Clearly $\phi\left(v_{n}(p)\right) \downarrow$ as $n \rightarrow \infty$. Put $\phi(p)$ $=\lim _{n=\infty} \phi\left(v_{n}(p)\right)$. Then $\phi(p)=1$ or 0.

Proof of 1). Let $p \in G$. Then clearly $D(\min (M, N(z, p)))=2 \pi M$ and $\mathfrak{M}(p)=1$ for $p \in G . \quad$ By definition $\left(\frac{1}{2 \pi M}\right) D\left(\omega\left(V_{M}(p), z, G\right)\right) \uparrow \mathfrak{M}(p)$ as $M \downarrow 0$, where $V_{M}(p)=E[z: N(z, p)>M]$. Hence for any given $\varepsilon>0$, there exists a number $M$ such that $\mathfrak{M}(p)<\left(\frac{1}{2 \pi M}\right) D\left(\omega\left(V_{M}(p), z, G\right)\right)+\varepsilon$ and we can find 
a compact set $K$ in $V_{M}(p)$ such that $D\left(\omega\left(V_{M}(p), z, G\right)\right)<D(M \omega(K, z))+2 \varepsilon$, because, if $F_{m} \uparrow F, \quad D\left(\omega\left(F_{m}, z, G\right)\right) \uparrow \quad D(\omega(F, z, G))^{[7]}$. Since $\delta\left(p, p_{i}\right) \rightarrow 0$ implies $N\left(z, p_{i}\right) \rightarrow N(z, p)$ in every compact set, we can find a number $i_{0}$ such that $V_{M-\varepsilon}\left(p_{i}\right) \supset K$ for $i \geqq i_{0}$, whence $\frac{D(\omega(K, z, G))}{2 \pi M} \leqq \frac{D\left(\omega\left(V_{M-\varepsilon}\left(p_{i}, z, G\right)\right)\right)}{2 \pi(M-\varepsilon)}$ $\leqq \mathfrak{M}\left(p_{i}\right): i \geqq i_{0} . \quad$ Let $\varepsilon \rightarrow 0 . \quad$ Then $\mathfrak{M}(p) \leqq \frac{\lim }{i} \mathfrak{M}\left(p_{i}\right)$.

Proof of 2). If $p \in G$, clearly ${ }_{v_{n}(p)} N(z, p)=N(z, p)$ and $\phi\left(v_{n}(p)\right)=1$ for every $n$. Put $g_{M}(p)=E\left[z:{ }_{v_{n}(p)} N(z, p)>M\right]$. Then by the definition of $\phi\left(v_{n}(p)\right)$, for any given $\varepsilon>0$, there exists a number $M_{0}<1$ such that $\phi\left(v_{n}(p)\right) \leqq$ $\frac{D\left(M \omega\left(g_{M}, z, G\right)\right)}{2 \pi}+\frac{\varepsilon}{2 \pi}=\frac{M}{2 \pi} D\left(\omega\left(g_{M}, z, G\right)\right)+\frac{\varepsilon}{2 \pi}$ for $M \leqq M_{0}$. Also we can find a compact set $K$ in $C g_{M}(p)$ such that $D\left(\omega\left(g_{M}(p), z, G\right)\right) \leqq D(\omega(K, z, G))+\varepsilon$.

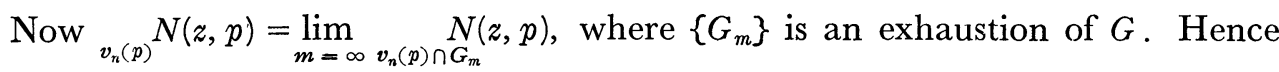
there exists a number $m_{0}$ such that

$$
{ }_{v_{n}(p)} N(z, p) \leqq \frac{M \varepsilon}{2}+\underset{v_{n}(p) \cap G_{m}}{N(z, p)} \quad \text { on } K \text { for } m \geqq m_{0} .
$$

Now $N(z, q)$ is continuous in $G-q$, whence $\underset{v_{n}(q) \cap G_{m}}{N(z, q)}$ is continuous on $K$ and there exists a number $i_{0}$ such that

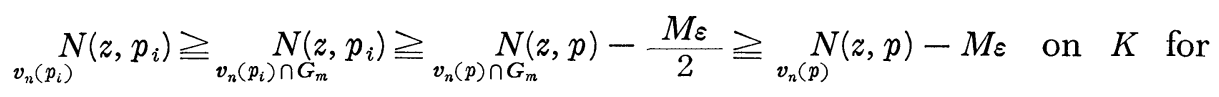

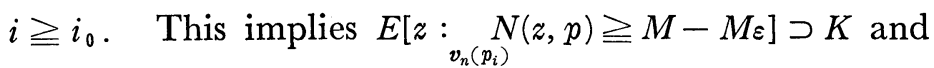

$$
D\left(\omega\left(g_{M-M \varepsilon}\left(p_{i}\right), z, G\right)\right) \geqq D(\omega(K, z, G)) \geqq D\left(\omega\left(g_{M}(p), z, G\right)\right)-\varepsilon \quad \text { for } i \geqq i_{0} .
$$

Thus $2 \pi \phi\left(v_{n}\left(p_{i}\right)\right) \geqq M(1-\varepsilon) D\left(\omega\left(g_{M-M \varepsilon}\left(p_{i}\right), z, G\right)\right) \geqq M D\left(\omega\left(g_{M}(p), z, G\right)\right)\left(\frac{M(1-\varepsilon)}{M}\right)$ $-M(1-\varepsilon) \varepsilon \geqq 2 \pi\left(\phi\left(v_{n}(p)\right)-\varepsilon\right)(1-\varepsilon)-M \varepsilon$ for $i \geqq i_{0}$.

Let $i \rightarrow \infty$ and then $\varepsilon \rightarrow 0$. Then $\frac{\lim _{i}}{i} \phi\left(v_{n}\left(p_{i}\right)\right) \geqq \phi\left(v_{n}(p)\right)$.

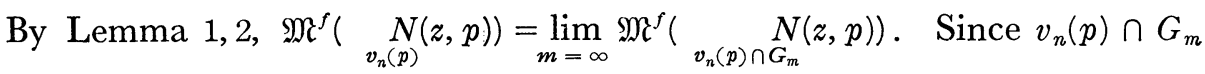
is compact, by the corollary of Theorem $2 \underset{v_{n}(p) \cap G_{m}}{N(z, p)}$ is representable by $\mu_{n, m}$ on $\overline{v_{n}(p) \cap G_{m}}$ with $\mathfrak{M i}^{f}\left(\underset{v_{n}(p) \cap G_{m}}{N}(z, p)\right)=\int d \mu_{n, m} . \quad$ Next $\left\{\mu_{n, m}\right\}$ has a weak limit $\mu_{n}$ as $m \rightarrow \infty$ such that $\mathfrak{M}^{f}\left(\underset{v_{n}(p)}{N(z, p))}=\int d \mu_{n}\right.$ on $\bar{v}_{n}(p)$. Let $n \rightarrow \infty$. Then $\left\{\mu_{n}\right\}$ has also a weak limit $\mu$ at $p=\underset{n>0}{\cap} \overline{v_{n}(p)}$ such that $\int d \mu=$

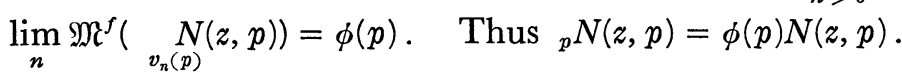


Case 1. $p \in G$. Then $\phi(p)=\lim _{n} \phi\left(v_{n}(p)\right)=1$.

Case 2. $\omega(p, z, G)>0$. In this case $\omega(p, z, G)=\lim _{n}{ }_{v_{n}(p)} \omega(p, z, G)=$ $\lim _{n} \int_{v_{n}(p)} N(z, p) d \mu(p)=K N(z, p)$. Now by $(9),{ }_{p} \omega(p, z, G)=\omega(p, z, G)$, i.e. $N(z, p)=$ ${ }_{p} N(z, p)$, whence $\phi(p)=1$.

Case 3. $\omega(p, z, G)=0 . \quad$ By $(8) \quad \phi(p) N(z, p)={ }_{p} N(z, p)={ }_{p}\left({ }_{p} N(z, p)\right)=$ $\phi^{2}(p) N(z, p)$. Hence $\phi(p)=0$ or 1 .

$N$-minimal function and $N$-minimal points. Let $U(z)$ be an $\mathbf{F}_{0} . \mathrm{S} . \mathrm{H}$. in $G$. If $V(z)=\lambda U(z): 0 \leqq \lambda \leqq 1$ for any F.S.H. $V(z)$ such that both $V(z)$ and $U(z)$ $-V(z)$ are F.S.H.s in $G$, we call $U(z)$ an $N$-minimal function. Then as the case that $\partial G$ is completely regular we have the following

Theorem 5.1).[7] Let $A$ be a closed set in $G+B$. Then $\omega(A, z, G)=$ $\int_{A} N(z, p) d \mu(p)$.

2). $\omega(p, z, G)=0$ for $p \in G$. If $\omega(p, z, G)>0, \omega(p, z, G)=K N(z, p)$ : $K>0$. We call such a point a singular point and denote by $B_{s}$ the set of singular points. By Theorem 2 we have

3). ${ }_{p} N(z, p)=\phi(p) N(z, p)$ and $\phi(p)=1$ for $p$ with $\omega(p, z, G)>0$ and $\phi(p)$ $=1$ or 0 . Denote by $B_{0}$ and $B_{1}$ sets of points of $B$ for which $\phi(p)=0$ and $\phi(p)=1$ respectively. Then by (2) $B_{s} \subset B_{1}$ and $B=B_{0}+B_{1}$.

4). $B_{0}$ is an $F_{\sigma}$ set of capacity zero, whence $B_{s} \subset B_{1}$.

5). If $U(z)=\int_{B_{0}} N(z, p) d \mu(p), \quad{ }_{B_{0}} U(z)=0$.

6). Let $U(z)$ be an $N$-minimal function such that $U(z)=\int_{A} N(z, p) d \mu(p)$. Then $U(z)=K N(z, p): p \in\left(G+B_{1}\right) \cap A$.

7). $\quad N(z, p)$ is $N$-minimal or not according as $\phi(p)=1$ or 0 .

8). Let $V_{M}(p)=E[z: N(z, p)>M]$ and suppose $p \in G+B_{1}$. Then $N(z, p)$

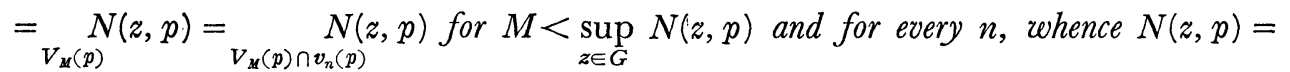
$M \omega\left(V_{M}(p), z, G\right)$ in $G-V_{M}(p)$.

9). Every potential $U(z)=\int N(z, p) d \mu(p)$ can be represented by another distribution $\mu$ on $G+B_{1}$ without any change of $U(z)$. This distribution is called canonical.

If $\partial G$ is completely regular $\mathfrak{M}^{f}(p)=1$ for $p \in G+B$. But in general cases $\mathfrak{M}(p)$ is not necessarily $=1$. We shall prove 
Lemma 4. $\quad \mathfrak{M}(p)=\mathfrak{M}^{f}(N(z, p))=1$ for $p \in G+B_{1}$.

Let $\left\{G_{m}\right\}$ be an exhaustion of $G$. By $p \in G+B_{1} \underset{v_{n}(p)}{N(z, p)}=N(z, p)$.

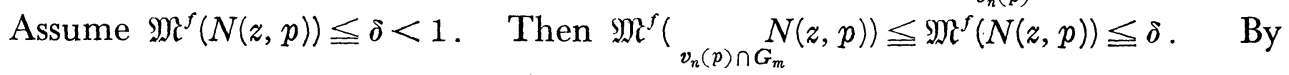
Theorem $2 \underset{v_{n}(\rho) \cap G_{m}}{N}(z, p)$ is represented by a mass $\mu_{n, m}$ on $\overline{v_{n}(p) \cap G_{m}}$ with $\int d \mu_{n, m} \leqq \delta$. Let $m \rightarrow \infty$ and then $n \rightarrow \infty$. Then ${ }_{p} N(z, p) \leqq \delta N(z, p)$. This contradicts ${ }_{p} N(z, p)=N(z, p)$. Hence $\mathfrak{M}(p)=1$.

THEOREM 6. Let $U(z)=\int_{G+B_{1}} N(z, p) d \mu(p)$. Then

$$
\mathfrak{M}^{f}(U(z))=\int d \mu
$$

where $U(z)$ is not necessarily an F.S.H. in G (clearly for an F.S.H. in G).

This is an extension of the corollary of Theorem 2.

Put $\phi(p, n, m)=\mathfrak{M}^{f}\left(_{v_{n}(p) \cap G_{m}}^{N(z, p))}\right.$. Then by Theorem 4 and by $p \in G+B_{1}$ $\phi(p, n, m) \uparrow \phi(p, n)=\mathfrak{M}^{f}\left({ }_{v_{n}(p)}^{N} N(z, p)\right)=\mathfrak{M}^{f}(N(z, p))=1 \quad$ as $m \rightarrow \infty . \quad$ Put $U_{m}(z)=\int_{v_{n}(p) \cap G_{m}} N(z, p) d \mu(p) . \quad$ Then

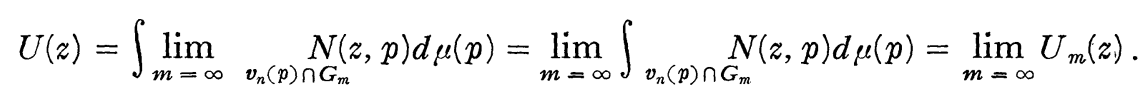

Now $\underset{v_{n}(p) \cap G_{m}}{N}(z, p)=\int_{\overline{v_{n}(p) \cap G_{m}}} N(z, q) d \mu_{p}(q)$ and since $\mu_{p}(q)>0$ only on a compact set $\bar{G}_{m}$, we have $\int d \mu_{p}(q)=\phi(p, n, m)$ by the corollary of Theorem 2. Hence $U_{m}(z)=\iint_{G_{m}} N(z, q) d \mu_{p}(q) d \mu(p)$ and $\mathfrak{M}^{f}\left(U_{m}(z)\right)=\int \phi(p, n, m) d \mu(p) . \quad$ It is easily verified that Lemma 1. 2. holds for F.S.H.s and $\mathfrak{M}^{f}\left(U_{m}(z)\right) \uparrow \mathfrak{M}^{f}(U(z))$, if $U_{m}(z) \uparrow U(z)$. Now $\mathfrak{M}^{f}\left(U_{m}(z)\right) \uparrow \mathfrak{M}^{f}(U(z))$ and $\phi(p, n, m) \uparrow \phi(p, n)=1$ as $m \rightarrow \infty$ for $p \in G+B_{1}$. Hence $\mathfrak{M}^{f}(U(z))=\int d \mu(p)$.

\section{REFERENCES}

[1] If $\partial G$ and $\partial D$ are compact and smooth, $d(\lambda, z)$ is given as $\frac{\partial N}{\partial n}(\zeta, z) d s$, where $N(\zeta, z)$ is the $N$-Green's function of $G-D$ with pole at $z$.

[2] Z. Kuramochi: Potentials on Riemann surfaces. Journ. Fac. Sci. Hokkaido Uni., XVI (1962). See page 14 of this paper.

[3] See [2].

[4] See [2]. 
[5] See [2].

[6] See [2].

[7] See [2].

[8] See [2]. 